\title{
DISEÑO DE UN ROBOT CARTESIANO PARA LA MEDICIÓN DE TEMPERATURA CORPORAL Y DETECCIÓN DE MASCARILLA FACIAL MEDIANTE SENSORES INFRARROJOS YVISIÓN ARTIFICIAL PARA PREVENIR EL CONTAGIO DEL COVID-19
}

\author{
DESIGN OF A CARTESIAN ROBOT FOR BODY TEMPERATURE MEASUREMENT \\ AND FACIAL MASK DETECTION USING INFRARED SENSORS AND ARTIFICIAL \\ VISION TO PREVENT THE SPREAD OF COVID-19
}

\author{
Sergio A. Gutiérrez Sánchez', Mario R. Hernández Valladares, \\ Diego A. Magallanes Chauca, Ricardo J. Palomares Orihuela
}

RECEPCIÓN: 24 DE AGOSTO DE 2021

ACEPTACIÓN: 10 DE SETIEMBRE DE 2021

\begin{abstract}
RESUMEN
Este proyecto tuvo por finalidad diseñar un robot cartesiano de un eje para la medición de la temperatura corporal y detección de mascarilla facial como parte del protocolo de control de acceso de personas en los centros comerciales de Lima, Perú. El robot cartesiano fue diseñado utilizando el software SolidWork y simulado mediante CoppeliaSim, mide la temperatura corporal a través de un sensor infrarrojo y detecta la presencia de mascarilla mediante una cámara GOPRO Hero 7 Black y una red neuronal convolucional. Mediante la prueba 1 se comprobó el funcionamiento del sistema de detección de mascarillas, al obtener la etiqueta "Con_Mascarilla" cuando la persona tiene puesta una. En la prueba 2 se simuló el desplazamiento del sensor infrarrojo y la medición de la temperatura corporal, que obtuvo una luz verde para una medición en el rango normal de $36^{\circ}$ a $37.5^{\circ}$. En la prueba 3 , se simuló el funcionamiento del robot al ingreso del centro comercial. La etapa final del proyecto incluye su implementación y validación mediante un sistema de alarma mediante voz.
\end{abstract}

Palabras clave: Robot cartesiano, visión artificial, red neuronal, Covid-19

\begin{abstract}
The objective of this project was to design a one-axis Cartesian robot for body temperature measurement and face mask detection as part of the access control protocol for people in shopping centers in Lima, Peru. The Cartesian robot was designed using SolidWork software and simulated using CoppeliaSim, it measures body temperature using an infrared sensor and detects the presence of a mask using a GOPRO Hero 7 Black camera and a convolutional neural network. Through test 1, the operation of the mask detection system was verified, obtaining the label "With_Mascarilla" when the person is wearing one. In test 2 , the displacement of the infrared sensor and the body temperature measurement were simulated, obtaining a green light for a measurement in the normal range of $36^{\circ}$ to 37.5 $\circ$. In test 3 , the operation of the robot at the entrance of the shopping center was simulated. The final stage of the project includes its implementation and validation through a voice alarm system.
\end{abstract}

Keywords: Cartesian robot, computer vision, neural network, Covid-19 


\section{INTRODUCCIÓN}

Debido a la pandemia por el virus Covid-19, las empresas se han visto obligadas a contratar personal adicional para el control en los ingresos de las personas en los establecimientos comerciales con el objetivo de medir la temperatura corporal y la verificación de la mascarilla facial. La labor del personal de control genera un gasto adicional mensual en la empresa y, además, pone en riesgo su salud al interactuar constantemente con otras personas. Esta situación ocasiona un incremento indirecto en la cantidad de contagiados por Covid-19, hecho que se vuelve crítico en épocas festivas. En estas se registra un incremento importante de casos confirmados como el de finales del ańo 2020 e inicios del año 2021 [1] vinculado con las festividades de Navidad y Año Nuevo. Este fue el inicio de la segunda ola con un incremento de 10,398 detecciones en la semana 51 del año 2020 a 29,744 detecciones en la segunda semana del año 2021.

Por ello, se buscó diseñar un robot cartesiano [2], [3] de 1 GDL que usa un sensor ultrasónico para obtener la altura de la persona que desea ingresar al centro comercial y en consecuencia controla el desplazamiento de un sensor infrarrojo hacia la muñeca de las personas para la posterior medición de su temperatura corporal. Asimismo, determina la presencia de la mascarilla facial mediante el análisis fotográfico de las personas. Este se realiza a través de una cámara y una red neuronal convolucional. De esta manera, en cuanto la temperatura corporal se encuentre en el rango permitido y la persona tenga colocada la mascarilla facial, el sistema autoriza su ingreso al centro comercial y abre la puerta de ingreso de manera automática; en caso contrario, se notifica a la persona que podría tener un problema de salud. Por otro lado, si la persona no colabora con las indicaciones del robot cartesiano, se procede a avisar por medio de una alarma al personal de seguridad del centro comercial, con la finalidad de evitar riesgos de contagio. Según el MINSA, en el mes de agosto, el Perú pudo superar la segunda ola tal como se muestra en la Figura 1. Para evitar en la medida de lo posible la llegada de una tercera ola, es necesario fortalecer los mecanismos de prevención de contagios.

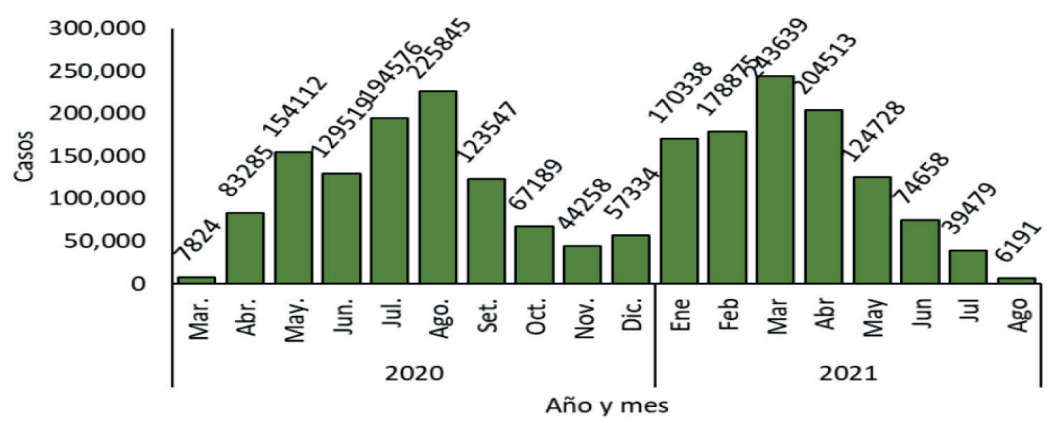

Figura 1. Casos confirmados de Covid-19, según fecha de inicio de sintomas Fuente: Sala Situacional Covid-19 Perú del MINSA

2 Estudiante del décimo ciclo de Ingeniería Mecatrónica de la Universidad Ricardo Palma. Cuenta con conocimientos en robótica e inteligencia artificial. Expositor en Student Poster Competition en la Conferencia Virtual LACCEI 2021. $<$ mario.hernandez@urp.edu.pe>

3 Estudiante del décimo ciclo de Ingeniería Mecatrónica de la Universidad Ricardo Palma. Cuenta con conocimientos en robótica, diseño gráfico y CAD. <diego.magallanes@urp.edu.pe>

4 Ingeniero Mecatrónico de la Universidad Nacional de Ingeniería, Doctor en Ciencias de la Educación. Magíster en Ciencias de la Educación con mención en Docencia Universitaria, Maestría en Ciencias de la Electrónica con mención en Ingeniería Biomédica. Docente de la Escuela Profesional de Ingeniería Mecatrónica de la Universidad Ricardo Palma. Senior Member IEEE. Miembro profesional RAS, EMBS, ComSoc y EduSoc - IEEE. Investigador Renacyt Carlos Monge IV. <rpalomares@ieee.org> 


\section{DISEÑO DEL SISTEMA DEL ROBOT CARTESIANO}

\subsection{Diseño mecánico del robot cartesiano}

El robot realiza un desplazamiento en el eje Z, como se observa en la figura 2, con la finalidad de mover el módulo de medición en función al $80 \%$ de la altura de la persona medida. Este procedimiento es controlado por una Raspberry Pi, con un sensor de ultrasonido [4] ubicado en el techo de la entrada del centro comercial. Después se realiza el desplazamiento, utilizando el módulo de medición, el cual toma la temperatura corporal mediante un sensor infrarrojo [5] y una cámara GOPRO Hero 7 Black; esta última detectará si el cliente porta o no una mascarilla facial. Si cumple los requisitos, se procede a abrir automáticamente las puertas del centro comercial.

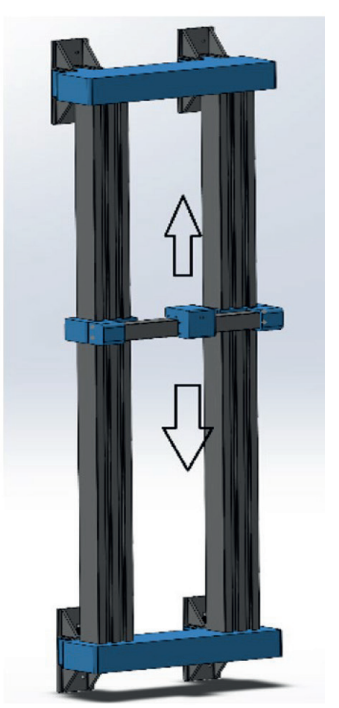

Figura 2. Estructura mecánica del robot cartesiano. Elaborado en SolidWork 2020 SP3.0

Con la señal que el sensor de proximidad detecte, se activan los motores conectados, como se aprecia en la Figura 3, a unos tornillos que giran mediante un acoplador hasta que el sensor infrarrojo esté a la altura requerida.

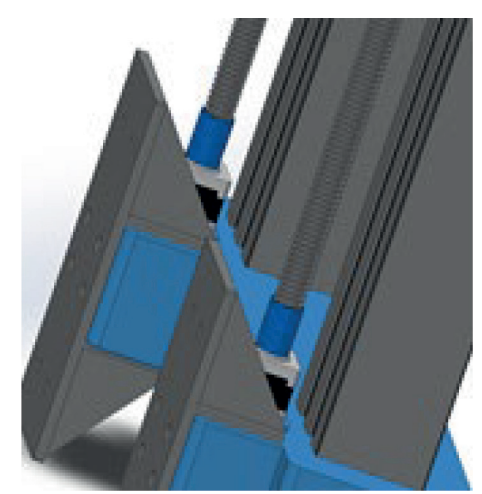

Figura 3. Motores paso a paso activados por sensor de proximidad. Elaborado en SolidWork 2020 SP3.0 
El robot diseñado en SolidWorks cuenta con una altura aproximada de 2 metros. Este tamaño permite que su uso incluya a personas con estatura por encima del promedio. Las medidas del robot cartesiano se aprecian en la figura 4.

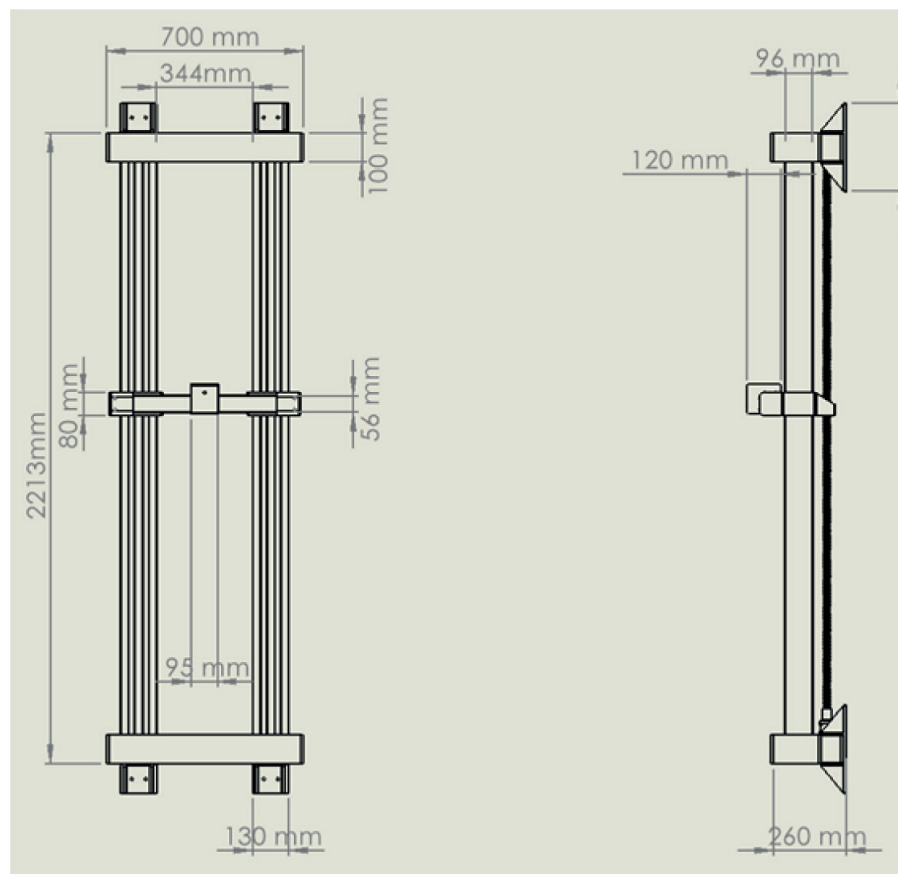

Figura 4. Dimensionado del Robot Cartesiano. Elaborado en SolidWork 2020 SP3.0

\subsubsection{Análisis cinemático y dinámico del robot cartesiano}

Se utilizó el algoritmo de Denavit-Hartenberg para calcular la cinemática directa del robot, tal como se observa en la figura 5.

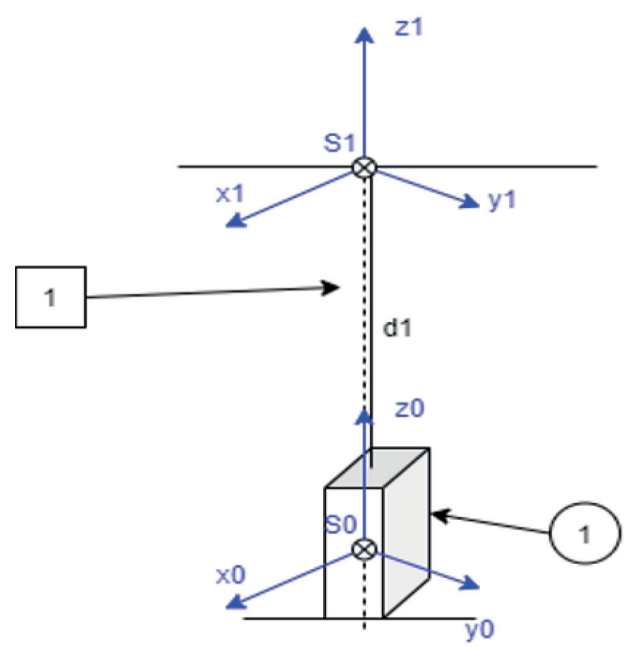

Figura 5. Esquema del robot cartesiano 
En la Tabla 1, se presentan los parámetros cinemáticos del robot cartesiano.

\begin{tabular}{|c|c|c|c|c|}
\hline Articulación & $\theta \mathrm{i}$ & di & ai & ai \\
\hline 1 & 0 & $\mathrm{~d} 1$ & 0 & 0 \\
\hline
\end{tabular}

Tabla 1. Parámetros Cinemáticos del robot

Donde:

$\mathrm{d} 1$ = desplazamiento variable del robot cartesiano

Luego se realiza el cálculo de la dinámica utilizando el método de Lagrange-Euler y se obtiene la ecuación 1:

$$
\mathrm{F} 1=m 1 *(\mathrm{~d} 1) \ddot{d}+9.8)
$$

Donde:

$$
\begin{aligned}
& \mathrm{F} 1=\text { fuerza ejercida por el motor } \\
& \mathrm{m} 1=\text { masa del módulo electrónico } \\
& \ddot{d} \quad=\text { segunda derivada del desplazamiento variable del robot cartesiano }
\end{aligned}
$$

\subsection{Diseño Electrónico del Robot Cartesiano}

Para el diseño electrónico, se describe el algoritmo que se implementó para la programación del sistema. El ciclo de funcionamiento del robot cartesiano inicia con la detección de la altura de la persona. Para tal fin, el módulo se posiciona al $80 \%$ de la altura de esta (de abajo hacia arriba). Después, mediante un algoritmo de Deep Learning, que utiliza una red neuronal convolucional, se identifica si la persona porta o no mascarilla facial. En caso de ser negativo, se prohíbe el ingreso y, en caso sea positivo, continúa con la medición de la temperatura corporal. Si la temperatura corporal de la persona se encuentra en el rango de $36^{\circ}$ a $37.5^{\circ} \mathrm{C}$ se apertura automáticamente la puerta de ingreso y se permite su acceso. En caso contrario, se muestra una señal que indique que no se le permitirá el ingreso, como se muestra en el diagrama de flujo de la figura 6.

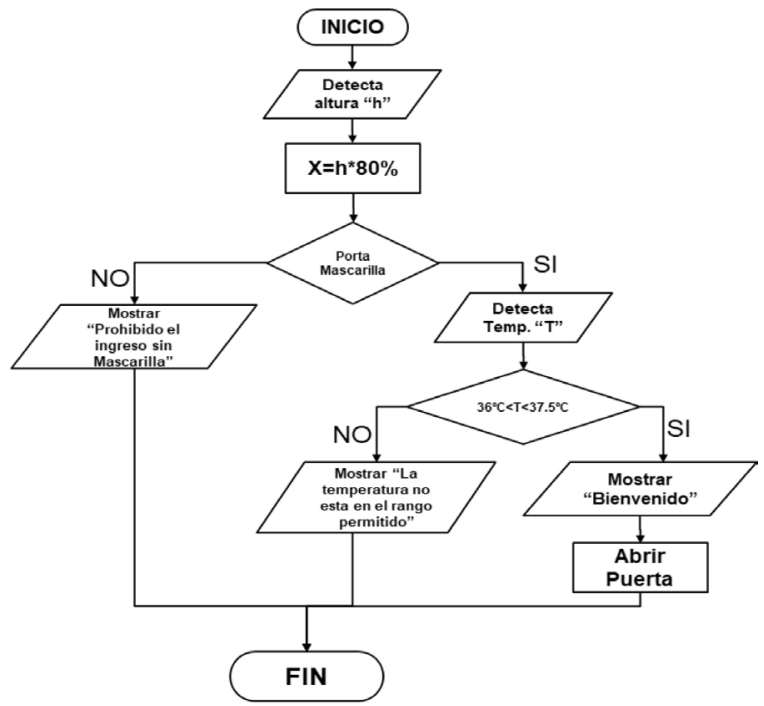

Figura 6. Diagrama de Flujo del Funcionamiento del Robot Cartesiano 
Para la simulación del circuito electrónico se utilizó Tinkercad, una plataforma de diseño para modelado 3D y circuitos electrónicos perteneciente a Autodesk. Como se observa en la figura 7, el circuito se simuló en la web para que se implemente en el robot cartesiano.

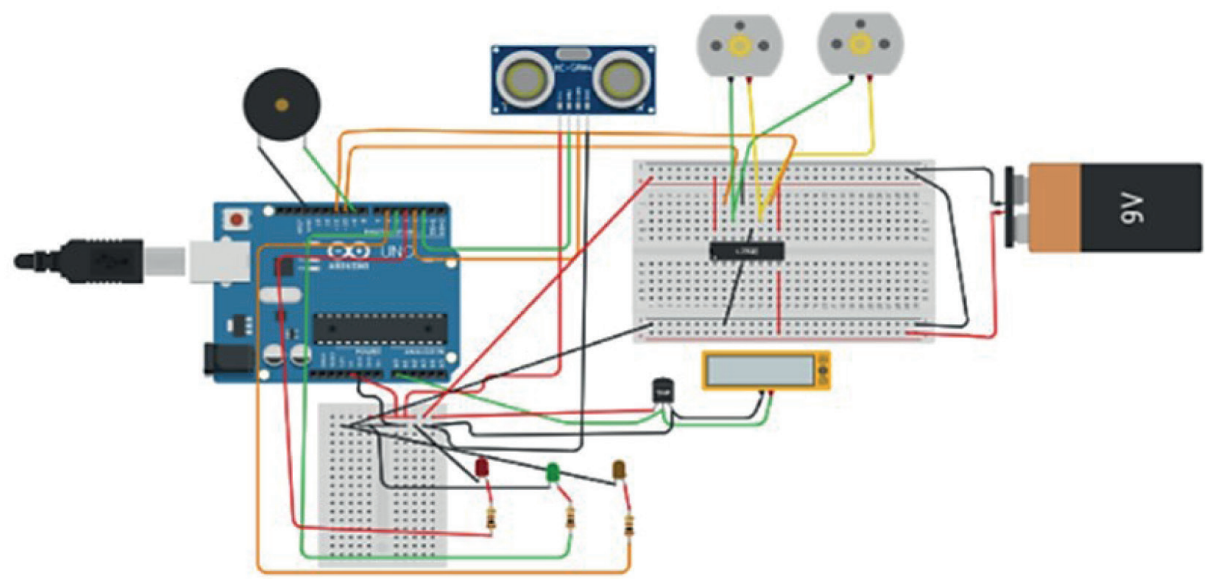

Figura 7. Partes del ensamblado electrónico. Elaborado en Tinkercad

En la figura 8, se observa el fragmento del código que permite determinar la presencia de alguien. En la línea 52, se calcula el tiempo en el cambio de estado mediante la función "pulseIn ()". En la línea 57, se determina la altura de la persona y se guarda en la variable "distancia". Si la altura es cero, el sistema no continúa; de lo contrario, el sistema detecta que hay una persona y sigue con el proceso.

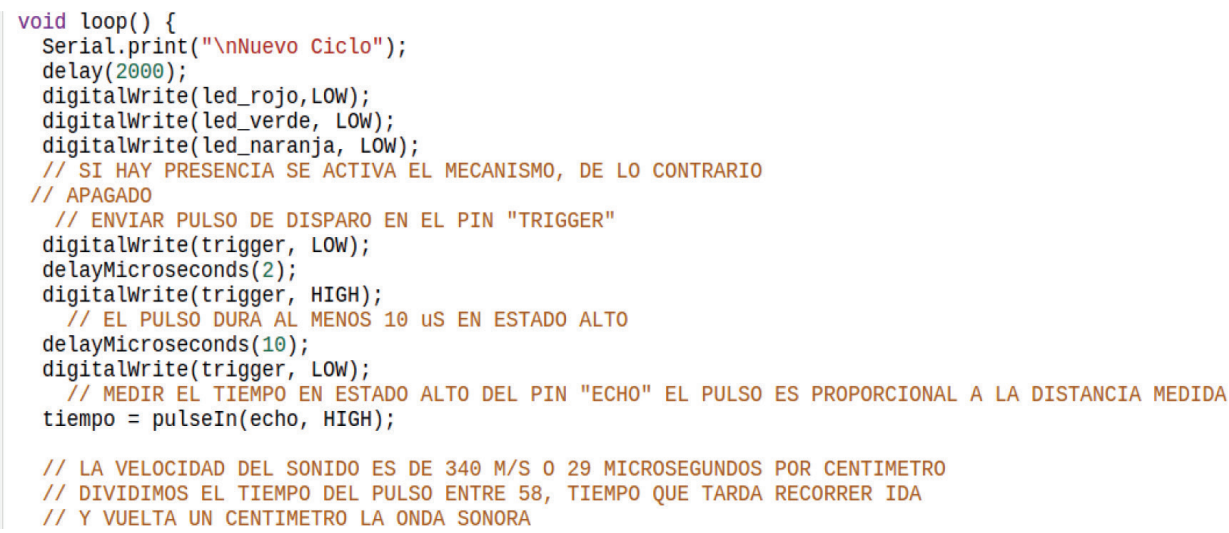

Figura 8. Fragmento del código utilizado para la simulación. Elaborado en Tinkercad

En la figura 9, se observa, en la línea 70, cómo se calcula la distancia de la persona en metros. En la línea 87, se guarda la señal analógica que retorna el sensor infrarrojo en la variable "tmpPin", simulado con un sensor tmp 36, para luego determinar la temperatura corporal de la persona mediante la fórmula de la línea 88 . Posteriormente, se prohíbe el ingreso de la persona si su temperatura corporal es superior a $37.5^{\circ} \mathrm{C}$, como se observa en la línea 92 . En caso contrario, se permite el acceso de la persona. 


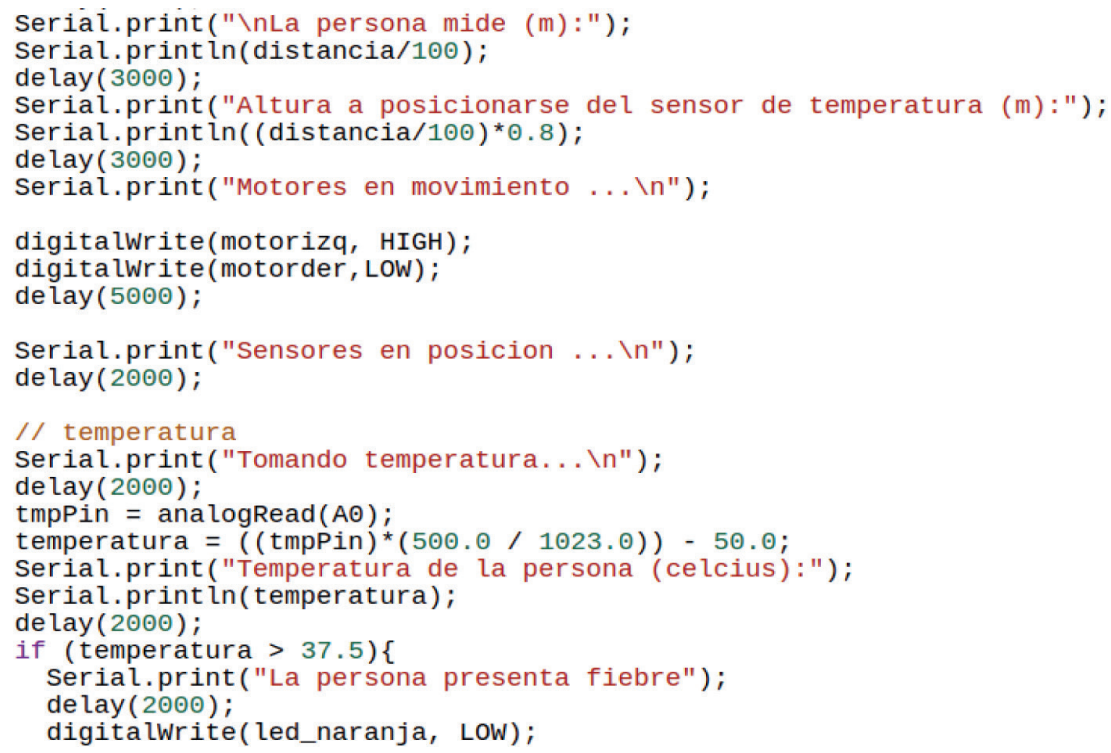

Figura 9. Fragmento del código utilizado para la simulación. Elaborado en Tinkercad

\subsection{Diseño del sistema de detección de mascarillas}

El desarrollo del sistema de detección de mascarilla facial se realizó en dos etapas: la detección de rostro y la detección de mascarilla facial. En la primera etapa, utilizando el software OpenCV, se captura en tiempo real el video proveniente de la cámara. Luego, mediante el módulo de Face Detection de MediaPipe [6], se procede a obtener el rostro de las personas que se encuentren en el campo de visión para luego procesarlos en la etapa de detección de mascarillas.

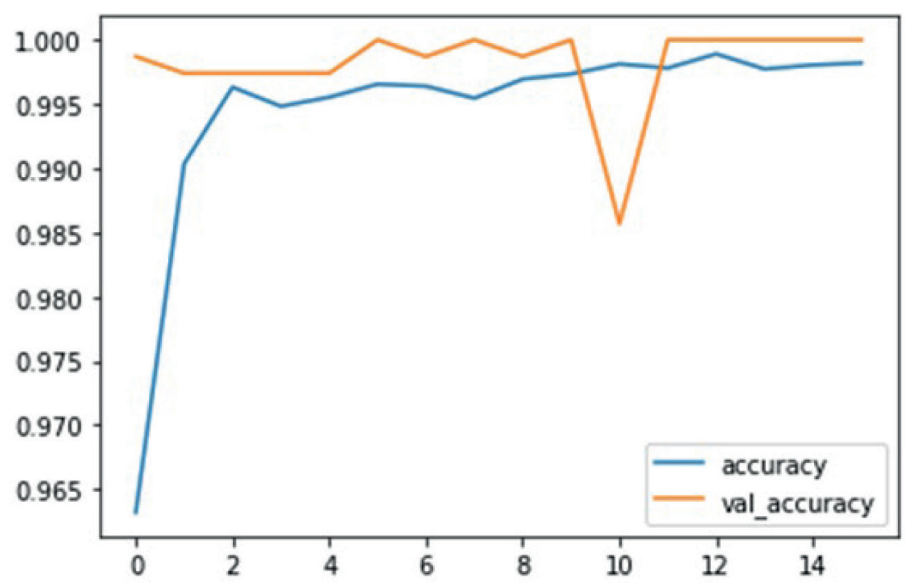

Figura 10. Gráfico de precisión vs épocas del entrenamiento

En la segunda etapa, se procede a reentrenar la red neuronal convolucional MobileNet [7], debido a que es un modelo eficiente y ligero por lo que se puede ejecutar con pocos recursos en la Raspberry Pi. Para la detección de mascarillas, se modificó la capa de entrada para que pueda procesar una ima- 
gen de 128x128 píxeles en formato RGB y la capa de salida para indicar si cuenta o no con mascarilla utilizando la función de activación Sigmoidal. Para el entrenamiento se utilizaron 12,800 imágenes y para la validación se utilizaron 790 imágenes, las cuales fueron obtenidas de Kaggle [8], [9] y se preprocesan al tamańo y formato concordante a la capa de entrada de la red neuronal convolucional. Se obtuvo un $99.82 \%$ de precisión como máximo con los datos de entrenamiento y un $100 \%$ de precisión como máximo con los datos de validación, los cuales se observan en la figura 10 .

Luego del entrenamiento, se procedió a implementar el modelo obtenido en el Sistema de Detección de Mascarillas. Las imágenes de los rostros obtenidos con MediaPipe son preprocesadas para cumplir con las condiciones de la capa de entrada de la red neuronal convolucional para identificar la presencia o no de las mascarillas. Esto se indica a través de un mensaje en la visualización de la imagen que cambia entre el color rojo y verde, el cual indica la ausencia o presencia de la mascarilla facial respectivamente. Para finalizar, envía el resultado al sistema principal del Robot Cartesiano para permitir o no el acceso y lo une al resultado de la temperatura corporal. El diagrama de flujo de datos del sistema de detección de mascarilla facial se observa en la figura 11.

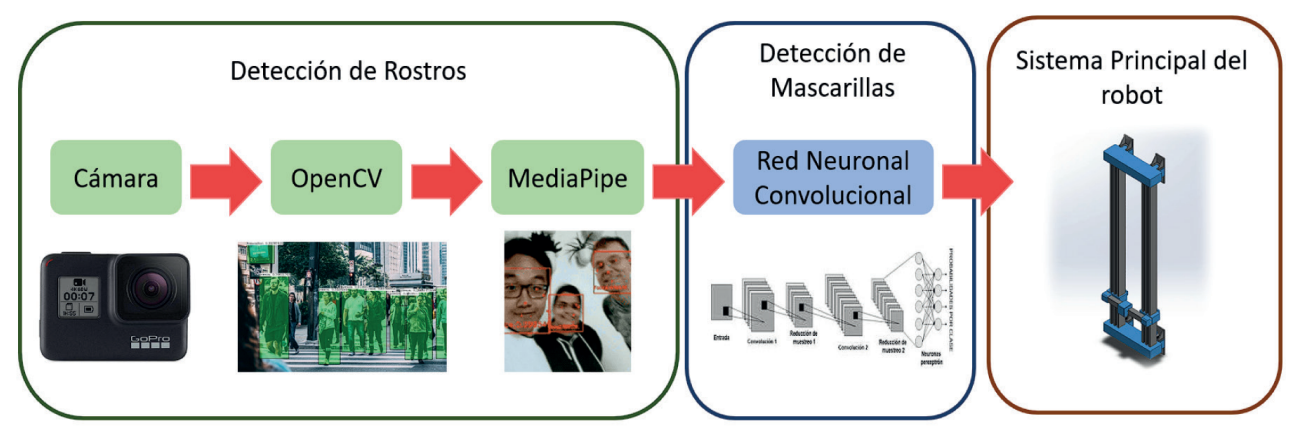

Figura 11. Diagrama de flujo de datos del Sistema de Detección de Mascarillas

\section{PRUEBAS Y RESULTADOS}

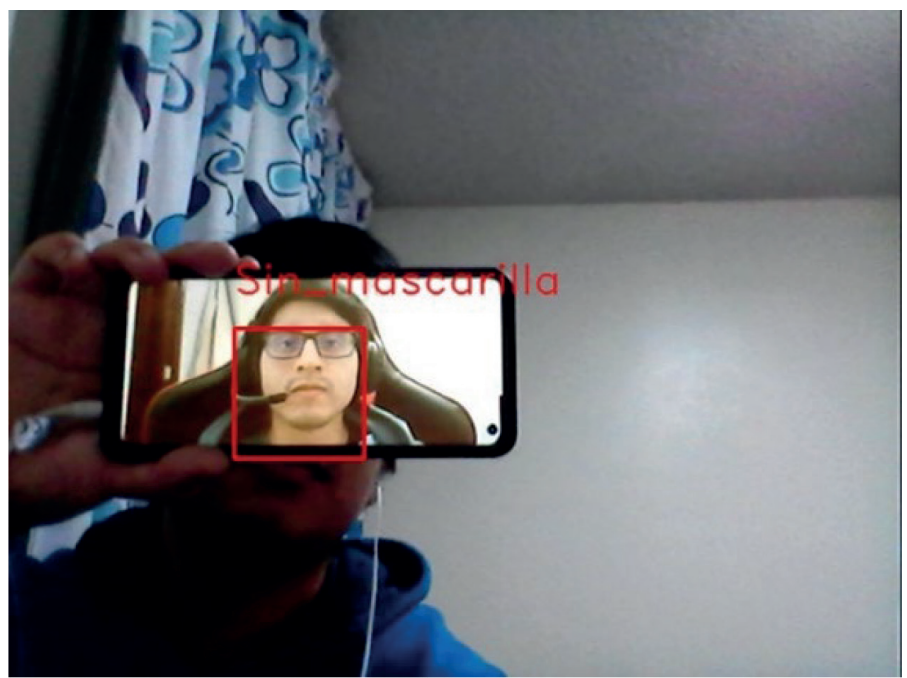

Figura 12. Detección de persona sin mascarilla 


\section{Prueba 1}

Se realizaron las pruebas del sistema de detección de mascarilla facial utilizando la IDE Spyder para su ejecución. Cuando la persona no posee la mascarilla facial, se observa un recuadro de color rojo y la etiqueta «Sin_mascarilla» encima del rostro como se muestra en la figura 12.

En caso contrario, el recuadro es de color verde y presenta la etiqueta «Con_mascarilla», tal como se muestra en la figura 13.

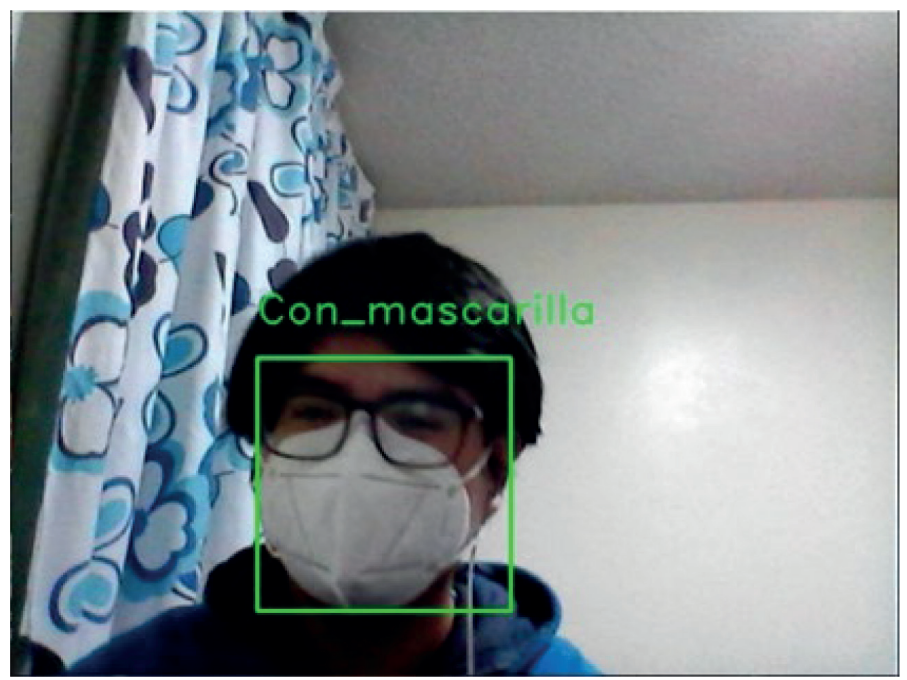

Figura 13. Detección de persona con mascarilla

\section{Prueba 2}

En esta prueba se simuló el posicionamiento del sensor infrarrojo y el ingreso mediante luces. Este arroja el color verde, que indica óptimo, cuando el sujeto está en el rango de $36^{\circ}$ a $37.5^{\circ} \mathrm{C}$; el color naranja si está en proceso; y rojo si está fuera de los parámetros normales. Estos resultados se muestran en las figuras 14,15 y 16 respectivamente.

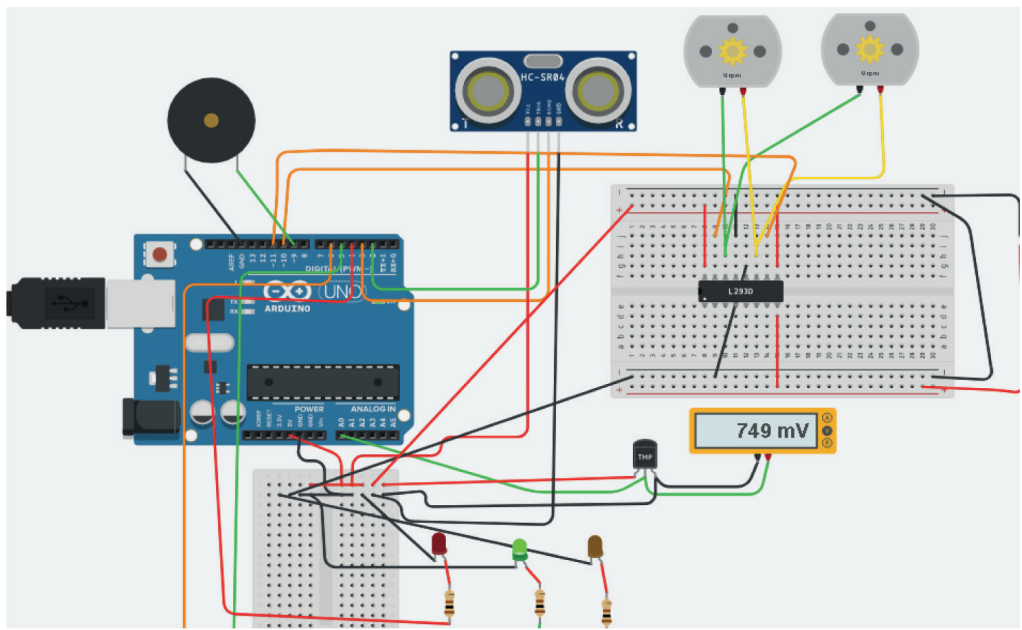

Figura 14. Señal de acceso permitido después de medición de temperatura. Elaborado en Tinkercad 


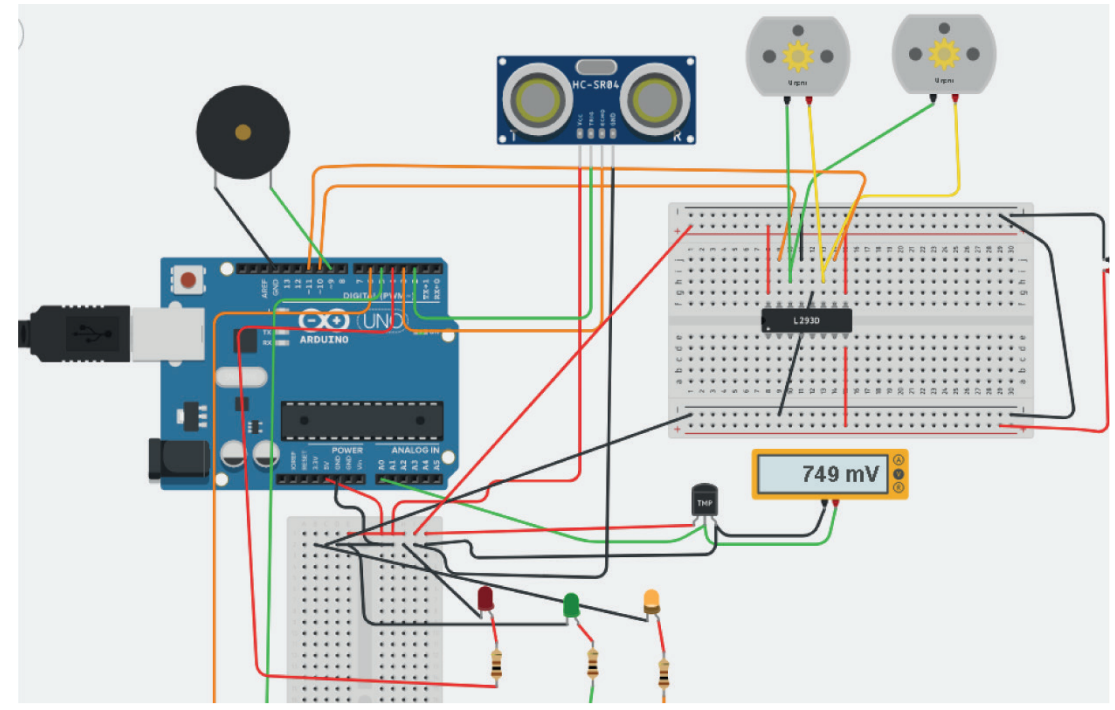

Figura 15. Señal de medición de temperatura en proceso elaborado en Tinkercad. Elaboración propia

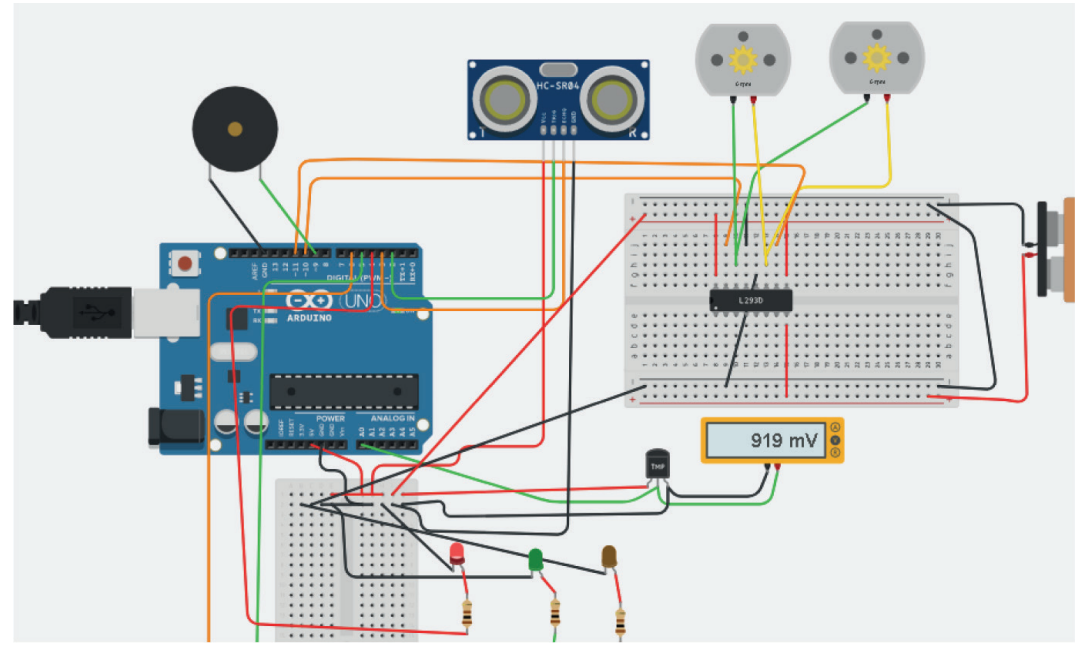

Figura 16. Señal de acceso denegado después de medición de temperatura elaborado en Tinkercad. Elaboración propia

\section{Prueba 3}

En esta prueba se realizó la simulación del robot cartesiano en el software CoppeliaSim. Primero se realizó la importación de los archivos stl del ensamblaje; luego se realizó la programación del sensor ultrasónico y de los motores para que, cuando se detecte a la persona, el sensor de ultrasonido mida su altura y luego realice el movimiento cartesiano al $80 \%$ de la medida obtenida para que mida la temperatura. En la figura 17, se observa el instante en el que la persona aún no llega al rango de detección del sensor de ultrasonido por la cual el robot se encontraba en su posición inicial. En la figura 18, se observa el instante en el que la persona fue detectada y el robot cartesiano se desplaza para realizar la medición de la temperatura corporal. 


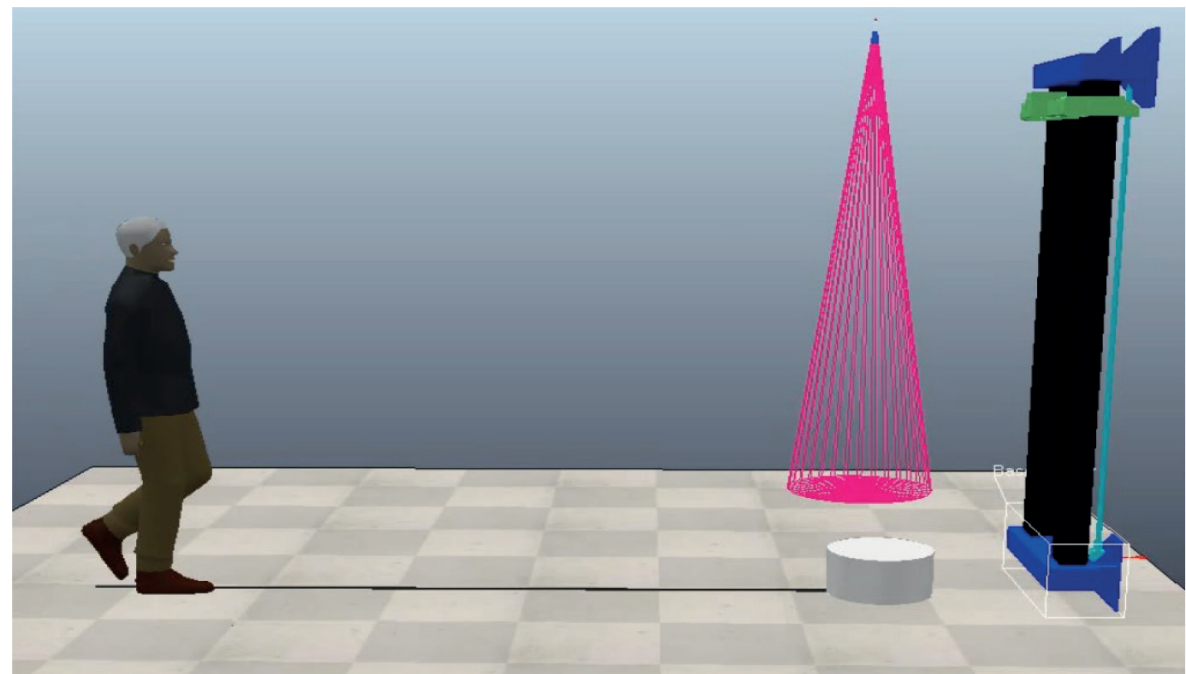

Figura 17. Simulación inicial del robot cartesiano. Elaborado en CoppeliaSim V4.2.0

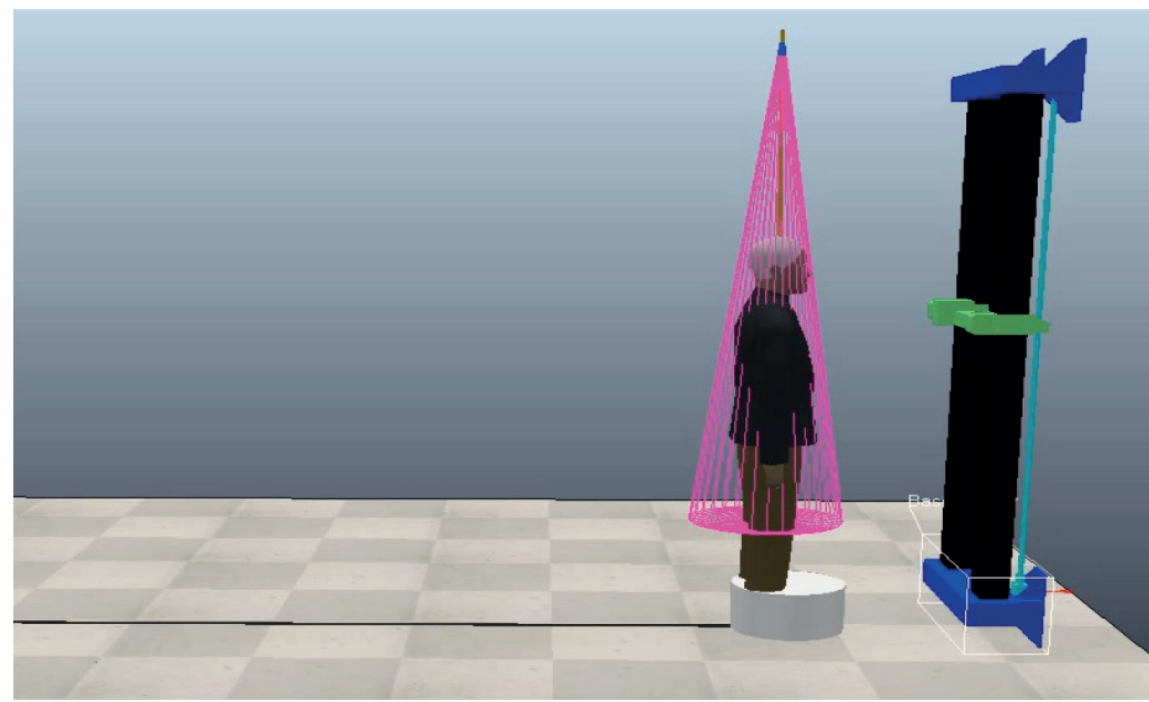

Figura 18. Simulación de persona detectada y desplazamiento del módulo de medición de temperatura corporal. Elaborado en CoppeliaSim V4.2.0

\section{CONCLUSIONES}

Para el diseño del robot cartesiano, se tuvo en consideración diversos tipos de soportes estructurales. Inicialmente, se evaluó empotrarlo en el suelo, sin embargo, debido a su altura de casi $2 \mathrm{~m}$ y su masa de aproximada de $200 \mathrm{~kg}$, presentaría un alto grado de inestabilidad, con el riesgo de caída, por lo que, se optó por empotrarlo a la pared a través de 04 soportes instalados en cada esquina.

En el diseño electrónico, se evaluó el uso de una variedad de motores DC y sensores, tanto de ultrasonido como infrarrojo, determinando el uso de aquellos modelos disponibles en el mercado 
local con la finalidad de contar, en el corto plazo, con un primer prototipo del robot cartesiano para su implementación y validación. [10]

Se observó durante las pruebas del sistema de detección de mascarilla facial que cuando una persona se tapa la boca con las manos o con algún objeto diferente a una mascarilla facial el sistema detecta que si tiene mascarilla, por lo que, en la segunda fase del proyecto, se adicionará fotografías de personas tapándose la cara en el dataset para que el sistema sea capaz de identificar estos casos adecuadamente.

Finalmente, en la simulación mediante el software CoppeliaSim, se tuvo que agregar un filtro de media exponencial a la medida obtenida con el sensor ultrasónico, de tal manera que el movimiento del robot cartesiano sea suave.

\section{REFERENCIAS}

[1] Sala Situacional Covid-19 Perú del MINSA (2021). Situación Actual Covid19 Perú 2020-2021. [En línea]. Disponible en: https://bit.ly/3mBWpcH_[Accedido: 29-dic-2021]

[2] R. Godoy y W. Rodríguez. "Diseńo y modelamiento de un robot cartesiano para el posicionamiento de piezas”, tesis de pregrado, Universidad de La Salle, 2007 [En línea]. Disponible en: https://ciencia.lasalle.edu.co/ing_automatizacion/50 [Accedido: 29-dic-2021]

[3] C. Rodríguez. "Diseño y construcción de un robot cartesiano con un control de posición de punto a punto", tesis de pregrado, Universidad Libre, 2017 [En línea]. Disponible en: https:// repository.unilibre.edu.co/handle/10901/11249 [Accedido: 29-dic-2021]

[4] "Sensor de ultrasonido HC-SR04". Naylamp Mechatronics, 2021. [En línea]. Disponible en: https://bit.ly/3qx1Meb_[Accedido: 29-dic-2021]

[5] "Sensor de temperatura infrarrojo". Naylamp Mechatronics, 2021. [En línea]. Disponible en: https://bit.ly/3qvt44R_[Accedido: 29-dic-2021]

[6] "MediaPipe Face Detection". MediaPipe, 2021. [En línea]. Disponible en: https://bit.ly/32LOP81 [Accedido: 29-dic-2021]

[7] I. Venkateswarlu, J. Kakarla y S. Prakash, "Face mask detection using MobileNet and Global Pooling Block", en 4th Conference on Information \& Communication Technology (CICT), 2020, pp. 1-5, doi: 10.1109/CICT51604.2020.9312083.

[8] “Face Mask Detection 12k Images Dataset". Kaggle, 2021. [En línea]. Disponible en: https://bit. ly/3EEdkRO [Accedido: 29-dic-2021]

[9] "Face Mask Detection Dataset". Kaggle, 2021. [En línea]. Disponible en: https://bit.ly/32JG8LN [Accedido: 29-dic-2021]

[10] R. Nope-Giraldo, L. Illapuma-Ccallo, R. Palomares, J. Cornejo y otros, "Mechatronic Systems Design of ROHNI-1: Hybrid Cyber-Human Medical Robot for COVID-19 Health Surveillance at Wholesale-Supermarket Entrances", 2021 Global Medical Engineering Physics Exchanges/Pan American Health Care Exchanges (GMEPE/PAHCE), 2021, pp. 1-7, doi: 10.1109/GMEPE/ PAHCE50215.2021.9434874. 